\title{
AMMU'S MAN: RECONNOITERING THE MACHISMO OF VELUTHA IN THE GOD OF SMALL THINGS
}

Soumen Mukherjee, Vellore Institute of Technology University, India, soumenprl@yahoo.co.in

Originalni naučni rad

DOI: 10.31902/fll.34.2021.2

UDC: 821.214.09-31

\begin{abstract}
Machismos, as is the instance with feminineness for women, are publically built gender profiles under which men are pigeonholed. The inferred affiliation between male bodies and machismos or masculinity presents us with an understanding of the sex/gender gap where 'sex' is seen as remaining a preeminence and 'gender' as a set of facets which are ancillary. New directions in feminist studies have begun to take up this issue of reconsidering or rediscovering masculinity, especially in the context of recent works of Literature. Arundhati Roy's Man-booker award winning novel, The God of Small Things (1997) is predominantly a novel about battle- in and through the body. Velutha, "The God of Loss. The God of Small Things" (Roy 265), whose name in Malayalam means "white" (Roy 73, 175 and 334), the colour affiliated both to sorrow and sunlit, has been depicted by the writer as the emblem of masculinity. Arundhati Roy builds an account that focuses on bodily happenstances that rebel considerable discourses and function as edges of cultural and social acquaintances. This study has been enthused by the comprehension that the subject of masculinity in women's writing has not yet been explored to that extent, which it was expected to be! Little attention has been given to the analysis of women's writing with the tools that theories of virilities provide. What masculinities emerge in Roy's The God of Small Things is the multi-layered, mongrel text, wavering between traversing valuations of indigenous acuities of the standing of the man, the bequest of interventionism, and the impresses of novelty and globalization! Hence, this study, not only reconnoiters the different traits of masculinity present in Velutha as envisioned by a female writer, but it also deliberates in detail the male identity construction.
\end{abstract}

Keywords: Machismo; Feminineness; Masculinity; Untouchable; Rebel; Sexuality; Rebel; Women's writing; Interventionism; Male Identity-construction

\section{Introduction}

Machismo is the public display of symptomatically masculine behavior; the distinction of being macho; male virility, masculine pride and a show of this gallantry. It is this strong or embroidered sense of masculinity stressing attributes such as physical valor, virility, and aggressiveness, which theorize supreme appraisal of physiognomies, culturally 
associated with the masculine and a vilification of individualities related with the feminine. ${ }^{1}$ The Spanish word "machismo" connotes ardent masculinity, primarily in males, though it can also be applied to women (e.g., marimacha). Some bases suggest its semantic roots to the uncouth Latin masclu, masculu, or masculus, from where we derived the word masculine or masculinity. As Raewyn Connell ${ }^{2}$ puts it, "Masculinity (is)...... the pattern or configuration of social practices linked to the position of men in the gender order, and socially distinguished from practices linked to the position of women". Nonetheless, 'Masculinity' like 'femininity' is an imaginary paradigm; because it conveys no connotation outside it's materialized and culturalized countenance! Masculinity studies predominantly became prevalent in the United States as a result of the feminist and gay deliverance movements of the $60 \mathrm{~s}$ and $70 \mathrm{~s}$. The emancipating language of the Civil Rights Movement stimulated these activities which wriggled to provide perceptibility to women and homosexuals. The first of these social activities, feminism, helped women to volte-face themselves. From this standpoint, feminism not only helped women to question outdated notions of femaleness, but also the sexual division per se. ${ }^{3}$ By demanding political and social changes, women were questioning patriarchal precepts and atrocity, and this would finally lead to the expansion of a critique of the hegemonic rules of gender. Similarly, the birth of the gay movement in 1969 led to the questioning of heterosexual normativity. ${ }^{4}$ Discerned against for a long time, gay men and lesbians integrated to fight for their cause, demanding the autonomy to choose one's sexual alignment as an unassailable right. Unruffled with feminism, the gay movement was thus formulating the new-fangled technique for the manifestation and the expansion of dissects of hegemonic/heterosexual masculinity. Alongside these two fundamental social movements, studies in men and masculinities originated as the third discipline having its stimulus from the Civil Rights Movement. It started garnering popularity in the United States during the late $50 \mathrm{~s}$ and became enormously vigorous in the subsequent era both in America and in other countries. Accessories in the crusade doomed racism and demanded racial equality between blacks and whites. As a number of scholars have argued, ${ }^{5}$ masculinity and whiteness are not only inter-reliant; they are amassed in such a manner that each category becomes more complex as the result of its association with the other. In contrast, by shielding non-whiteness, the Civil Rights Movement was, consequently, also tiling the way for an egalitarian society. 


\section{Masculinity studies within the ambit of gender readings}

Masculinity studies, drawing on feminist theory, accentuated and magnified the analysis of masculinity within gender studies. Much of the current scholarship about gender has absorbed itself on the role of women who are molded as symbols of the nation and "mother" land. Thus, more often than not, the women's bodies become locate of contesting culture, tradition and the nation. ${ }^{6}$ It must be noted in this regard that since the late $60 \mathrm{~s}$ and early $70 \mathrm{~s}$, women's studies have created most of the vocabulary for academic deliberations dealing with variation and the construction of gender-that is, the cultural constructions of femininity as well as that of masculinity (Sangeeta Roy 134). Nevertheless, of late, investigating this gendered version has thrown- up new questions on the role of masculinity and especially the male body. Accordingly, academic work on masculinity has industrialized across a number of disciplines, which has often progressed in dialogue with feminist politics, sharing forebodings regarding the dogmata of gender discourses and critiquing the power relations that are vociferous in a male-controlled civilization. Even so, it should be implicit that the use of the terms male (sex) and machismo /masculinity (gender) are sticky. ${ }^{7}$ The notions of gender and masculinities are multifarious in their edifices and embedded in hierarchal, historical, and cultural meanings. ${ }^{8}$

The delineations of gender and masculinity are again barbed, grounded on the nodes of period, class, race, ability, and sexual orientation to the extent that Connell and Messerschmidt ${ }^{9}$ have opined that machismo or "masculinities are configurations or practice that are accomplished in social action and, therefore, can differ according to the gender relations in a particular social setting" (836). Masculinity is thus a set of behaviours, prospects and body related / psychological traits that is constructed as inherent to manhood, i.e. XY chromosomes and male sexual features. Canaan and Christine Griffin ${ }^{10}$ therefore urged that women's libber consequently must be even more resolute about conducting research on men and masculinity at a time when a growing number of men are beginning to conduct apparently comparable research. Masculinity studies span old-fashioned punitive distributes; therefore, it is an interdisciplinary field of traditional, societal, ancient, mental, financial, and inventive analysis that cross-examines the edifices of virility in cultures across the world and in olden times. Moreover, it gazes at the edgy and multifaceted relationship between hegemonic masculinities, i.e., the idea of a "real man" in a given time and place and subsidiary virilities, i.e. masculinities that, in a given time and place, fall short of the "real man" idyllic. For example, being involved in sports and 
being a benefactor are the examples of hegemonic masculinity, while shunning sports and being a stay-at-home-dad are examples of subsidiary masculinities. ${ }^{11}$ Thus, scholars of masculinity often refer to machismos in the plural to highlight the diversity of meanings, roles, and behaviors consumed in the term.

\section{Masculinity studies in the realm of English Literature}

In place of a sensible or insensible imaginary or prognosis of other worlds, literature can divulge features of masculinity that might not come out or be noticeable in daily life or in other types of ethnic artifacts. As Stefan Horlacher ${ }^{12}$ professes, literature and masculinity go hand in hand. Although it is accurate that film, painting, sculpture, performance art, and music channel question masculinity and while it is also precise that literature is in no way the only transmitter of gendered symbol, literary form unavoidably yields its own exclusive depiction of masculinity. In the literature on masculinities, appraisals of masculinity and clarifications of the relations between masculinity/ masculinities and those people defined as 'men' vary according to notional belvedere. For example, in accounts drawing on the natural sciences, masculinity/ masculinities are the result of physiological factors, such as hormones or chromosomes. In contrast, from the more critical, academic perspective of Literature or the social sciences, machismo or masculinities are understood as a form of power relation, both among men themselves and between men and women. It is solely for this motive alone that fictional scrutiny in the twenty-first century institutes a decisive and vivacious extension of masculinity studies.

Extensive publication of imperative works in literary criticism with the birth of the study of masculinity in the 1980s can be pigeonholed as largely non-literary in nature, with the social sciences taking the most palpable lead in what was then a new and sometimes argumentative approach to gender. Notwithstanding the fact that gender is often experienced as fervently subtle-a core façade of our uniqueness-masculinities are produced and reproduced through the course of our daily interactions as well as within the larger institutions of society. In place of essentialism, masculinities are argued to arise from the social contexts in which men live, for example, from their positions in the various institutions and organizations of their society and/or in the context of the socially available discourses about gender. In nearly all cases, questions of identity, whether cultural or individual are central to masculinity studies, meaning that approaches to fleshand-blood human beings and approaches to literary sit-ins are not fully distinct. Sociological or anthropological considerations of masculinity 
can be and were in many ways familiarized to literary studies: fabulous propositions of masculinity may legalize formations of gender in the social sciences, but literariness may also transmogrify such outsets in ways that only take place within the deceptive transcription. Machismo or Masculinity Studies (or, as it is also often called, Men's Studies) in English Literature owes to feminism an enormous intellectual and political debt. In fact, it would not have existed without feminism and its courage to question patriarchal power and privilege. Men's studies work with feminist studies to question the relationship that men have with macho power throughout different chronological and antique times.

\section{The birth of "men's studies"}

As a moderately new-fangled arena of study, men's studies were fashioned largely in response to, and as an assessment of, an emerging men's rights movement, and as such, has been taught in academic locales only since the $1970 \mathrm{~s} .{ }^{13}$ Within the ambit of masculinity, Men's studies are grounded in men's activities and the common goal, shared with feminists, of creating a partnership in the gender framework of the 1970s and challenging accepted notions of masculinity and femininity. Yet feminist and men's studies did not always share common epistemological concerns, as there was a constant possibility of cooptation and misuse of feminist knowledge. ${ }^{14}$ In the 1990s, the renewed hegemonic masculinity was integrated into men's studies, and today the epistemic background is still not without ambivalence, which is a sign of the transition of 'masculinities' of the 1980s and is frequently responding, directly or indirectly, to the idea that masculinity was natural or essential. In the social sciences, it was responding to sex- role theory, in which the male sex role was taken as an unvarying, unwavering, and normative alignment. In his pioneering book The Myth of Masculinity, the psychologist Joseph Pleck explained that there was at the time no systematic formulation of the male sex role identity paradigm (MSRI). ${ }^{15}$ Although, his book aimed to critique that approach to gender, he provided an all-inclusive overview of the archetype later. On the contrary, studying up in gender research - that is, studying the superordinate category "men" - is still occasionally met with resistance from Feminist scholars, who have long made the important and valid critique that nearly all knowledge production, not explicitly labeled feminist, has implicitly studied men. ${ }^{16}$ Some argued that studying subordinated groups is obligatory for working towards egalitarianism and human liberation, while studying men re-centers men's experiences, draws attention and resources away from women, and thereby supports the male supremacist status quo. Nonetheless, 
feminist scholars and activists are increasingly recognizing the importance of addressing or including men (Casey \& Smith 232-36), since, Men's Studies scholars do not believe in pursuing investigation into masculine turf alone. Instead, they try to collaborate with feminists and scholars of race, class, and sexuality in seeking multifarious inquiries about the ways in which humanity creates and reins us as sexed and gendered individuals.

Outmoded scholarship used to speak of "sex differences," where behavioral differences were usually thought of as correlative with distinctive, and biological differences between women and men to be unvarying. Against this background, the women's studies scholarship which grew out of the contemporary feminist movement, insisted that the biological distinction male/female was not equivalent to or necessarily correlative with the social distinction masculine/feminine, and popularized the category of "sex roles" to speak of social/cultural differences between women and men. ${ }^{17}$ Most were willing to draw the clear political implications of this framework that if sex roles were learned or acquired, rather than innate and given, then they could also be unlearned and changed. Over time, however, problems emerged with the "sex role" framework. As the sex role hypothesis became radicalized, as more and more facets of femininity and masculinity came to be conceptualized as merely "roles," any remaining notion of a vital, natural self- extinct entity amidst the array of social roles being played upon. What emerged from this development was that new directions in feminist studies have begun to take up this issue of reconsidering or revitalizing masculinity, especially in the context of recent works of Literature.

\section{Machismo or masculinity in the context of The God of small Things}

One of the most important areas of research in gender and postcolonial studies is the analysis of indigenous masculinities within colonial contexts. This contour of exploration foregrounds the gendered, race and class dynamics of colonialism and nationalism and also provides opportunities for alternative gender practices that challenge hegemonic structures of white, middle-class patriarchy. For a better understanding of masculinity from the perspective of a woman writer, we may take into consideration; Arundhati Roy's much celebrated Man-booker award winning novel, The God of Small Things ${ }^{18}$, which is predominantly a novel about battle- in and through the body. Velutha, "The God of Loss. The God of Small Things" (265), has been depicted by Roy as the emblem of machismo or masculinity, even though he belongs to the class of so-called untouchables in the caste- 
divided Indian society. Among the male downtrodden characters, Velutha, the younger son of Vellya Paapen is the real central character of the novel, as he is self-illumined and has got the self-esteem-the qualities which make a man outstanding.

Over the years and across numerous contexts, men's bodies have become important sites where masculinity has been played out. According to Freud, the human subject has always been sexed, and that despite the biological differences, males and females have become particular social subjects. ${ }^{19}$ This biological individual can be viewed as a blank canvas upon which gendered identities are projected and performed through socialization. Therefore, the supposed differences between men and women are accentuated through the legitimization of social stereotypes. Roy's novel focuses on Velutha's muscular body and his near nudity, which rather than reifying him, depicts a specific kind of allure, which may be time and again, sexual in nature. Renowned feminist writer Sujala Singh discovers that Velutha's body in the novel is "described obsessively". ${ }^{20}$

Indeed, Roy's depiction of Velutha's body is undoubtedly having its sexual delineation, since he is often bare-chested, dressed only in a mundu. Kant, in this regard argued that "sexual love makes of the loved person an object of appetite; as soon as that appetite has been stilled, the person is cast aside as one casts away a lemon which has been sucked dry." ${ }^{21}$ In other words, looking at a nude person filled us (of course from the perspective of the opposite sex) with carnal desire, and that yearning induces a form of mind sightlessness. Instead of seeing the individual as having agency, he or she becomes a means to an end, nothing but a pitcher for the opposite gender's gratification (in most of the cases). Kant was describing a phenomenon known as objectification, in which seeing a body turns the entire person into a physical object.

\section{Sexual dominance vs. sexual submissiveness $\&$ racism}

All through these years, the social meaning of sex (gender) is created by sexual objectification of women whereby women are viewed and treated as objects for satisfying men's desires. ${ }^{22}$ Hence, masculinity is often seen as sexual dominance, femininity as sexual compliance within the ambit of a typical patriarchal society. Nonetheless, Roy's portrayal of Velutha and Ammu's erotic liason and the representation of the marginalized character's body are tied -in entirely with a different feminine sensibility. The text includes detailed descriptions of Velutha's physical beauty, as perceived by a woman writer through the eyes of her female protagonist, Ammu: 
"She saw ridges of muscle on Velutha's stomach grow taught and rise under his skin like the divisions on a slab of chocolate. She wondered how his body had changed-so quietly from a flat muscled boy's body into a man's body. Contoured and hard. A swimmer's body. A swimmer carpenter's body. Polished with high-wax body polish" (Roy 175).

The novelist is successful in describing the physical delineation of a bare-chested "Dark Man", the lasting impressions of which had its interminable influence on Ammu. Indians have varied degrees of color as well as facial features based on the geographical area to which they belong. Since the significance of skin colour in the Indian sub-continent is very paramount, the repetitive references to Velutha's 'black' body are particularly worthy of analysis. As Amali Philips ${ }^{23}$ explains: "The concept of skin-colour in India and more generally in South-Asia, embraces much more than chromatic qualities, for the semantics of colour include cultural perceptions and judgments about associated moral and behavioural qualities, health and appearance, and individual and collective identities" (253), whereas, "the social perception of the inferiority of dark skin is reinforced by its association with the lower classes in the region: dark skin also evokes the physical ravages of toiling 'long in the hot sun' "(355).

It is important to mention here a few instances from ancient India that show the mention of dark skinned 'heroes'; therefore, one can assume that at the time the color black was acceptable as a skin color of heroes, some of the most powerful gods and goddesses, and beautiful princesses and therefore, it is unlikely that people of the time saw being black as a depraved thing. Hindu ancient texts show that Krishna was the dark hero of Yadava tribe. The word Krishna itself means 'black' in Sanskrit. ${ }^{24}$ Similarly, the Rig Veda's hero Trasadasyu, son of Purukutsa, is the dark-complexioned leader of the other dark Dasyus. ${ }^{25}$ However, internalized racism within Indian society shows strong links with the notion of a color complex. Physical beauty, then, is closely tied in with social hierarchies and, in this cultural context is very much colour coded. Ammu's lover's skin is 'so black' that his parents named him 'Velutha', meaning 'White' in Malyalam (Roy 73) Indeed, Arundhati Roy seems to make a cognizant effort to insist on the beauty of Velutha's blacknessof his 'dark legs and smooth ebony chest' (334-5). Thus, the novel seems to endorse the hard reality that the biased view of Indian history was in keeping with 'nineteenth century European theories about racial evolution and made the further conquest of India by the British seem inevitable \& beneficial" (Tickel 25). 
Roy's characterization of Velutha could also, perhaps, lend itself to criticisms of gratuitous eroticization of the working-class male body. Velutha's schooling and his subsequent professional training of carpentry by a Carpenter's Guild in Bavaria for a period of three years, radically changes his awareness from the old sense of life to new ideas and progressive sense. It is mainly for this reason that Ammu attributes her lover's muscular beauty to his occupation as a carpenter:

"As she watched him, she understood the quality of his beauty. How his labour had shaped him. How the wood he fashioned had fashioned him. Each plank he planned, each nail he drove, each thing he made, had moulded him. Had left its stamp on him. Had given him his strength, his supple grace" (Roy 334).

Whereas middle class, professional men are more likely to exert power through the adroit use of modern technology, men in laborintensive, semi-skilled and trained occupations are more likely to express power physically. It is overbearing that "where men are economically dependent on the sale of their labour, the expression of maleness provides a means to exert power". ${ }^{26}$ In his essay on the eroticization of the working class in pornography, Stephen Donaldson ${ }^{27}$ contends that the psychological source of the attraction that the working classes exert on the elite comes from the perception that the upper classes have lost their masculine vitality. Men from the leisured classes are seen to be "removed from the exercise of physical power, while the (young) males of the lower class are more robust, earthy, grounded, more in touch with their sexuality, more physically aggressive, in short, more macho" (Roy 145). While it is difficult to deny that the subaltern's body in the text bears the stamp of his physical labour, it is important to note that the portrayal of Velutha's masculinity does not conform to stereotypical notions of a working-class masculinity that is overly aggressive and macho. On the contrary, in the final lovemaking scene, it is Ammu who initiates physical intimacy with him:

"She went to him and laid the length of her body against his. He just stood there. He didn't touch her.... She unbuttoned her shirt. They stood there. Skin to skin...she pulled his head towards her and kissed his mouth. A cloudy kiss. A kiss that demanded a kiss-back." (Roy 335)

Hence, more than the male, it is the female who initiates the gamut of love-making by instigating the enamored progression of hugging \& smooching; and here lies the success of Roy in depicting the sexual dominance of the woman over the sexual submission of the man! 


\section{Depiction of masculine sexuality from a feminine perspective}

Human sexuality has many aspects. In biology, sexuality describes the reproductive mechanism and the basic biological drive that exists in all sexually reproducing species and can encompass sexual intercourse and sexual contact in all its forms. There are also emotional and physical aspects of sexuality. ${ }^{28}$ These relate to the bond between individuals of the opposite sex, which may be expressed through profound feelings or emotions. Sexual rights are a fundamental element of human rights. They encompass the right to experience pleasurable sexuality, which is essential in and of itself and, at the same time, is a fundamental vehicle of communication and love between people. Sexual rights $^{29}$ include the right to liberty and autonomy in the responsible exercise of sexuality (HERA 27). The highly erotic depiction of the sexual encounter between Ammu and Velutha is referred to throughout the novel and serves as the whirlwind from place to place which the chronicle portrays and it becomes an apt manifestation of sexual right. Only currently in the end, under a chapter figuratively authorized "The Cost of Living," the erotica between Velutha and Ammu reaches its crowning. The novelist pronounces the volatile sexualintercourse in enthralling modus, which depicts in a new-fangled manner of a man's body being sexually explored by a female paramour:

"Ammu, naked now, crouched over Velutha, her mouth on his. He drew her hair around them like a tent ... She slid further down, introducing herself to the rest of him. His neck. His nipples. His chocolate stomach. She sipped the last of the river from the hollow of his navel. She pressed the heat of his erection against her eyelids. She tasted him; salty in her mouth ... She felt his belly tighten under her, hard as board. She felt her wetness slipping on his skin...took her nipple in his mouth and cradled her other breast in his calloused palm. Velvet gloved in sand paper ... At the moment that she guided him into her, she caught a passing glimpse of his youth, his youngness, the wonder in his eyes at the secret he had unearthed and she similes down at him as though he was her child." (Roy 336)

While the description seemingly moves away from the gender issues by undermining masculinity in the frame of sexual contravention, Sheila Jeffreys ${ }^{30}$ illustrates the distinction between sexual liberalism and sexual libertarianism when she writes:

"Sexual liberals are those who subscribe to the 1960s agenda of sexual tolerance, to the idea that sex is necessarily good and positive, and that censorship is a bad thing. Sexual libertarians have 
a more modern agenda and actively advocate the outer fringes of sexuality, such as sadomasochism, with the belief that sexual minorities are at the forefront of creating the sexual revolution'” (15).

The fact that they lovers in the story become invested with "feminine" traits redirects the focus towards the gender issue. This emasculation of male becomes a pre-requisite for them to join a mode of misdemeanor, which in this case belongs to the female realm as a potential site of power struggles. The policemen, who tortured Velutha, make fun of him for being a homosexual or transvestite because of his painted nails. The construction of the narrative extends this notion of the feminine as a central expression of alteration, but also of métier and longing, to the depiction of the body. While in a way glorifying the female body in all its possibilities and potential, Roy mocks the male body in its attempt to convey power that it does not really possess. If the political body is inevitably masculine, as critics have argued, then the writer, by mocking the masculine body, is also undermining its power and norm as the pattern to be followed in a patriarchal society. The author produces narratives that open venues to possible destabilizing readings of issues of gender and demands of physical pleasure, as Velutha's transformation from boyhood to manhood gratifies Ammu. Thus, her mind is preoccupied with Velutha, who is a representative of machismo or masculine vigor, where she initiates to love him, not amicably but bodily. Ammu craves for this man fervently and aches for him with the whole of her biology against the established norms of patriarchy, where only a male has the unlimited right to think of gratifying his sexual appetite.

\section{Conclusion}

Masculinity has long been a comparatively impervious social category, receiving petite consideration as a focus of study in its own right. But social scientific scholarship on men and masculinities has grown exponentially within the past few decades. In thinking of "masculinity-as-enactment" it must be recollected that those who do not accomplish their masculinity in a culturally approved manner are liable to be ostracized, even penalized. At one level, this is born of the recognition that early feminist and gender theory focused almost exclusively on the position and experience of women. Men, except where they were situated as part of the problem, were neither the object nor the subject of study. This changed in Anthropology when the cultural construction of masculinity became the focus of enquiry, with 
'male' and 'female' emerging as complex and changing symbolic categories. Recent theoretical developments have challenged stereotypes by showing how myriad masculinities are produced within specific historic and cultural domains, and have replaced monolithic notions of men's power by demonstrating that men wield and access power differently, depending on their racial, class and sexual locations. Most men are still culturally propelled to integrate dominance, whether in terms of crude physical strength or displays of masculine rationality and competence, into their presentation of self, which in reality has been challenged by Roy in her novel. Of course, by presenting Velutha's naked \& muscular beauty, the archetype that holds that masculinity and femininity are restraints into which all biological males and females are inevitably trimmed, begins to be sternly destabilized. It proved that "a Man" can also be displayed by a female writer as an object of sexual satisfaction and voluptuous fulfillment! In the present research, male body, sexualities and societal interfaces provide the foundation for appreciating the ethnic and momentous edifices of machismo or ardent masculinity. It also brands itself accessible as a new trend in feminists' readings, by means of which a variety of established settings can be altered. The God of Small Things pretty well fits into a feminist text, foregrounding many imperceptible obstacles patriarchy has continued to raise in women's way to gain parity with men. Susheila Nasta is very apt in describing the new mission like this:

"The post-colonial woman writer is not only involved in making herself heard, in changing the architecture of male-centered ideologies and languages, or in discovering new forms and language to express her experience, she has also to subvert and demythologize indigenous male writings and traditions which seek to label her." 29

To conclude, in the ongoing investigation, we have detected that Ammu's recognition of Velutha as her "Man" is an impetuous issue full of valor in suffragette terms, because in a society where proclamation of feminine sexual yearning outside the indorsed conjugal frame and wife-hood is challenging, Ammu takes the drastic step. In averring her own "biological" craving for a man who is the emblem of machismo, it appears that Ammu endeavors an insurrection of the male tendency to dominate by being, inevitably, the initiator of the sexual act. Hence, while reconnoitering the machismo or masculine traits of Velutha in her novel, Roy on the one hand, tries to underpin a new-fangled system of gender norms that reprieves all men with the process of identity 
construction, while on the other, affords equal acclaim to feminist pursuits in salvaging her mind and body!

\section{Works Cited}

"Definition of MACHISMO." Merriam-webster.com. N.p., 2020. Web. 27 Nov. 2020.

Connell, R. W., and James W. Messerschmidt. "Hegemonic Masculinity." Gender \& Society 19.6 (2005): 829-859. Web.

Nash, Margaret. "Gender Trouble: Feminism and the Subversion of Identity. By Judith Butler. New York: Routledge, 1990. - Homophobia: A Weapon of Sexism. By Suzanne Pharr. Inverness, CA: Chardon Press, 1988." Hypatia 5.3 (1990): 171-175. Print.

Fingrutd, M. "Book Reviews: Barry D. Adam, The Rise of a Gay and Lesbian Movement. Boston: Twayne Publishers, 1987, Pp. 203." International Journal of Comparative Sociology 28.3-4 (1987): 248-249. Print.

DiPiero, Thomas. White Men Aren't. Durham: Duke University Press, 2002. Print.

Chatterjee, Indrani. "En-Gendering India: Woman and Nation in Colonial and Postcolonial Narratives. By Sangeeta Ray. Durham, N.C.: Duke University Press, 2000. Viii, 198." The Journal of Asian Studies 60.2 (2001): 591-593. Print.

Collinson, David L., and Jeff Hearn. "Men as Managers, Managers as Men: Critical Perspectives on Men, Masculinities and Managements." Capital \& Class 22.2 (1998): 191-193. Web.

Faulkner, Gary L., Michael S. Kimmel, and Michael A. Messner. "Men's Lives." Contemporary Sociology 19.3 (1990): 351. Print.

Connell, R. W., and James W. Messerschmidt. "Hegemonic Masculinity." Gender \& Society 19.6 (2005): 829-859. Web.

Connell, R.W. "Book Reviews: MEN, MASCULINITIES AND SOCIAL THEORY. Edited by Jeff Hearn And David Morgan. London, Unwin Hyman, 1990. 252 Pp. (Paper)." The Australian and New Zealand Journal of Sociology 28.2 (1992): 277-278. Web.

Carrigan, Tim, Bob Connell, and John Lee. "Toward A New Sociology of Masculinity." Theory and Society 14.5 (1985): 551-604. Print.

Funk, Wolfgang. "Stefan Horlacher (Ed.). Constructions of Masculinity In British Literature From The Middle Ages To The Present." Anglia 131.4 (2013): n. pag. Print.

Allan, Jonathan A. "Phallic Affect, Or Why Men's Rights Activists Have Feelings." Men and Masculinities 19.1 (2015): 22-41. Print.

Patterson, Lesley. "Plural Masculinities - The Remaking of the self In Private Life20102sofia Aboim. Plural Masculinities - The Remaking of the self In Private Life. Aldershot: Ashgate 2010. 198 Pp. (Hardback), ISBN: 9780754674672." Gender in Management: An International Journal 25.6 (2010): 524-527. Web. 
Hoffnung, Michele. "Book Reviews." Psychology of Women Quarterly 9.1 (1985): 161-162. Print.

Casey, Erin, and Tyler Smith. "“How Can I Not?": Men's Pathways to Involvement in Anti-Violence Against Women Work." Violence against Women 16.8 (2010): 953-973. Print.

Goldsmid, P. L. "Masculine/Feminine or Human? An Overview of the Sociology of Sex Roles. By Janet Saltzman Chafetz. Itasca, III.: Peacock, 1974." Social Forces 54.3 (1976): 729-730. Print.

Roy, Arundhati. The God of Small Things. Compass Press: London, 1998. Print.

Buker, Eloise A. "Feminism and Philosophy: Perspectives on Difference and Equality. Moira Gatens. Bloomington: Indiana University Press, 1991." Hypatia 10.2 (1995): 171-173. Print.

Singh, Sujala. "Postcolonial children representing the nation in Arundhati Roy, Bapsi Sidhwa and Shyaam Selvadurai." Wasafiri 19.41 (2004): 13-18. Print.

Kant, Immanuel, and Roger J. Sullivan. "Kant: The Metaphysics of Morals." (1996): Print.

Eisenstein, Zillah. "Toward a Feminist Theory of the State. By Catherine A. MacKinnon. Cambridge: Harvard University Press, 1989. 320p. \$25.00." American Political Science Review 84.2 (1990): 635-637. Print.

Philips, Amali. "Gendering Colour: Identity, Femininity and Marriage in Kerala." Anthropologica 46.2 (2004): 253. Print.

Kanta, Surya. Sanskrit-Hindi-English dictionary. International Publication Service, 1975. Print.

Sutherland, Sally J. "Śūdras in Ancient India: A Social History of the Lower Order Down to Circa A.D. 600. By Ram Sharan Sharma. 2nd rev. ed. New Delhi: Motilal Banarsidass, 1980. xix, 344 pp. Appendixes, Bibliography, Index. Rs 90 (cloth)." The Journal of Asian Studies 42.4 (1983): 1004-1005. Print.

Kelly, Michael J. "Book Reviews: Contemporary Perspectives on Masculinity: Men, Women, and Politics in Modern Society. By Kenneth C. Clatterbaugh. Boulder, CO: Westview Press, 1990, 182 pp., \$34.95 (hardbound), \$14.95 (paperback)." Affilia 6.3 (1991): 99-99. Print.

Buss, David M., and David P. Schmitt. "Sexual Strategies Theory: An evolutionary perspective on human mating." Psychological Review 100.2 (1993): 204-232. Print.

Cavendish, Marshall. "Sex and Society." Retrieved June 21, 2017. The Term Human Sexuality Broadly Refers to How People Experience and Express Themselves as Sexual- Beings, 2, 2010, 384.Print.

"HERA." International Women's Health Coalition. N.p., n.d. Web. 27 Nov. 2020.

Acorn, Annalise. "The Sexual Liberals and the Attack on Feminism by Dorchen Leidholdt \& Jance G. Raymond, eds." Alberta Law Review (1969): 518. Print.

Hull, Akasha. "Motherlands: Black Women's Writing from Africa, the Caribbean and South Asia. Susheila Nasta Moorings and Metaphors: Figures of Culture and Gender in Black Women's Literature. Karla F. C. Holloway." Signs: Journal of Women in Culture and Society 19.3 (1994): 762-765. Print. 
আম্মুর পুরুষ : 'The God of small Things এ ভেলুথার পৌরুষ এর অন্নেষণ

সমাজে নারীদের যেরকম ভাবে নারীত্বের মাধ্যমে সনাক্ত করা হয়ে থাকে, ঠিক সেই ভাবেই পুরুষদেরও তাদের পৌরুষের দ্বারা শ্রেণীবদ্ধ করা হয়/ ঠিক যেমন পৌরুষ বা পুরুষত্ব দিয়ে পুরুষদের চিন্নিত করা আমাদের সমাজব্যবস্থায় লিঙ্গ বৈষম্য বোঝাতে সাহায্য করে, ঠিক সেইরকম ভাবেই নারী-পুরুষের এই লিঙ্গ ভেদাভেদিই আমাদের 'gender সংক্রান্ত বিষয়ের গভীরে প্রবেশ করতে উৎসাহ যোগায় / নারীবাদী অধ্যয়নের ক্ষেত্রে, বিশেষত সাহিত্যের সাম্প্রতিক রচনার প্রসঙ্গে, পুংলিঙ্গ অন্নেষণ বা পুনরায় আবিষ্কারের বিষয়টি, একটি নতুন সংযোজনা। অরুন্ধতী রায়ের ম্যান-বুকার অ্যাওয়ার্ড বিজয়ী উপন্যাস, The God of small Things (১৯৯১) মূলত দেহের উথ্যান-পতন কে নিয়ে লেখা একটি গল্প । ভেলুথা, "The God of Loss. The God of Small Things" (রায় ২ 26৫), যার নামের মালায়ালামে অর্থ "সাদা" (রায় 73৩, ১5৫ এবং ৩৩৪), যেটি জীবনের অন্ধকার এবং আলো উভয়েরই সাথে যুক্ত, সেটিকে লেখিকা পৌরুষের প্রতীক হিসাবে চিত্রিত করেছেন। অরুন্ধতী রায় এমন একটি উপন্যাস লিখেছেন, যেখানে শরীর তার ভাষা দিয়ে কথা বলে এবং সাংস্কৃতিক ও সামাজিক পরিচিতির মাধ্যম হিসাবে কাজ করে। তবে প্রত্যাশা মতন মহিলাদের লেখায় পুরুষের যৌনতার বিষয়টি এখনও সেই পরিমাণে অনুসন্ধান করা হয়ে ওঠেনি, যা প্রত্যাশিত ছিল! আর এখানেই এই গবেশোনার সার্থকতা/আসলে পৌরুষের যৌনতা বা যৌন আকর্ষণ করার ক্ষমতা মূলক তত্ত্বগুলি নিয়ে মহিলাদের হস্তক্ষেপ এবং লেখার বিশ্লেষণের দিকে খুব কম মনোযোগ দেওয়া হয়েছে। রায়ের ‘God of small Things'- এ পৌরুষত্বের বিভিন্ন -স্তরের আলোচনা করা হয়েছে/একজন পুরুষমানুষের দেশীয় অভিনবত্ব ও গোঁড়ামির মাঝে দোদুল্যমান থেকে বিশ্বায়নের চিন্তাধারায় নিজেকে সঁপে দেয়ার মধ্যে যে নতুনত্ব থাকে, তাকেই চিত্রায়িত করা হয়েছে! সুতরাং, এই অধ্যয়নটি কেবল নারী লেখকের কল্পনা অনুসারে ভেলুথার পৌরুষকে উন্মোচন করে না, বরঞ্চ এটি পুরুষের পরিচয় নির্মাণের বিষয়টিকেও বিস্তারিতভাবে অন্নেষণ করে।

মূল-শব্দ: পৌরুষ ; নারীত্ব; পৌরুষত্ব; অস্পৃশ্য; বিদ্রোহী; যৌনতা; মহিলাদের লেখা; হস্তক্ষেপ; পুরুষ পরিচয়-নির্মাণ 